\title{
CONTACT TRANSMISSION OF WAVE MOTION BETWEEN TWO SOLIDS WITH AN INITIAL GAP
}

\author{
J. R. BARBER \\ Department of Mechanical Engineering and Applied Mechanics. University of Michigan, Ann Arbor, \\ MI 48109. U.S.A. \\ maria Comninou \\ Department of Civil Enaineering, University of Michion, Ann Arbor, MI 48109, U.S.A. \\ and \\ John Dundurs \\ Department of Civil Engineering, Northwestern University, Evanston, IL 60201, U.S.A.
}

(Received 24 August 1981)

\begin{abstract}
Amtract-The paper treats the trannission of wave motion from one solid to another when the bodies are initially separied by a small ap. If a pulse of elastic waves strikes the surface of one of the solids and the amplitude of the pulse is large enough, the solids can come into contact and interact locally. The dynamic interaction is treated using Coulomb's friction law, and a method is presented for finding the slip and stick zones in the contact reion when the incidence ande of the pulse is subcritical. Unusual results are encountered for large friction coeficients.
\end{abstract}

\section{INTRODUCTION}

In treating the reflection and transmission of elastic harmonic waves between two solids that can separate, it is discovered that the solids must be pressed together for a continuing interaction[1]. If this is not done, the solids move apart during the early stages of the process, and a continuously maintained train of incident waves sees a free surface. The early part of the interaction can be studied, however, by analyzing a pulse of finite extent. In such cases it is not required that the solids be pressed together and, in fact, interaction is possible even if there is small initial gap between them.

In a previous paper [2], a solution was given for the problem of an elastic pulse striking the frictional interface between two half spaces and producing localized regions of separation and slip between the bodies. In this paper we consider the related problem for which a localized region of contact between two otherwise separated bodies propagates along the interface due to the action of an incident pulse in one of them. The problem is illustrated in Fig. 1. The two half spaces are initially separated by a uniform gap $d$ and the upper solid has a tangential velocity $u$ to the right as shown. A $P$ or $S V$ pulse strikes the interface at an angle of incidence $\theta_{0}$ and produces $P$ and $S V$ pulses reflected at angles $\theta_{1}, \theta_{2}$. If some interaction occurs between the

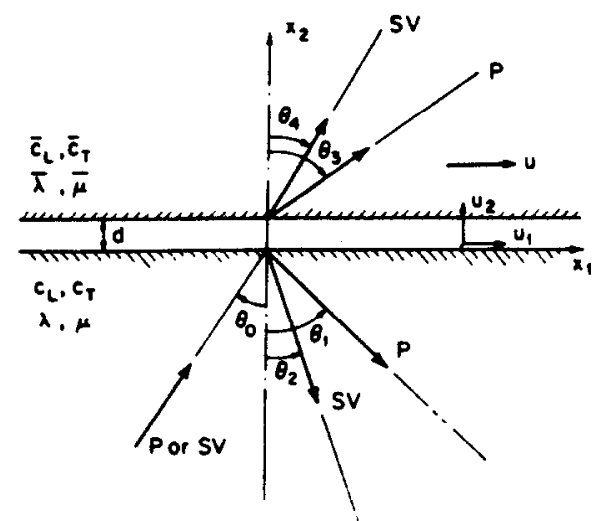

Fig. I. Incident, reflected and refracted waves at the interface with an initial gap $d$. 
bodies, we also anticipate the development of refracted $P$ and $S V$ pulses in the upper solid at angles $\theta_{3}, \theta_{4}$ respectively.

The velocities of $P$ and $S V$ waves are denoted by $c_{L}, c_{T}$ respectively and we use bars to distinguish the properties of the upper body. The angles are related by the equation

$$
\frac{\sin \theta_{0}}{c_{0}}=\frac{\sin \theta_{1}}{c_{L}}=\frac{\sin \theta_{2}}{c_{T}}=\frac{\sin \theta_{3}}{\bar{c}_{L}}=\frac{\sin \theta_{4}}{\bar{c}_{T}}
$$

and the disturbance due to the pulse propagates to the right along the surface of the lower body at velocity

$$
v=c_{0} / \sin \theta_{0} .
$$

As in [2], we restrict attention to those values of $\theta_{0}$ for which $v$ is supersonic with respect to both solids, i.e. eqn (1) defines real values for all the angles $\theta_{i}$.

\section{METHOD OF SOLUTION}

As in [2], we solve the problem by superposition of two parts, but here the initial solution corresponds to the situation where there is no interaction between the half spaces, i.e. we find the reflected pulses on the assumption that the surface of the lower body is free of tractions. This is a classical problem [3] and need not be considered here. The pulse will cause local displacements $u_{1}, u_{2}$ at the free surface which are stationary with respect to the dimensionless moving co-ordinate

$$
\eta=k_{0}\left(x_{1} \sin \theta_{0}-c_{0} t\right)
$$

The wave number $k_{0}$ can be regarded as the reciprocal of a characteristic length for the pulse. In other words, a bulge or depression runs along the free surface at velocity $v$, its shape being described by the displacement $u_{2}(\eta)$.

If the maximum height of this bulge is less than $d$, the free surface solution is the final solution of the problem and the half spaces do not interact. However, the interesting case is that in which contact occurs. A corrective solution must then be superposed to satisfy the physical conditions of the problem and eliminate the interpenetration predicted by the free surface solution alone.

The gap between the bodies is

$$
g_{0}(\eta)=d-u_{2}(\eta)
$$

and we define the gap opening velocity for the free surface solution

$$
G_{0}(\eta)=\frac{\partial g_{0}}{\partial t}=-c_{0} k_{0} \frac{\partial g_{0}}{\partial \eta}
$$

There is also a corresponding slip velocity

$$
H_{0}(\eta)=u-\frac{\partial u_{1}}{\partial t} .
$$

A positive value of $H_{0}$ corresponds to the upper body slipping to the right over the lower body.

\section{THE CORRECTIVE SOLUTION}

In the corrective solution, equal and opposite tractions are applied to the two surfaces to satisfy appropriate conditions on displacements in contact zones. This solution is developed in [2] from the results for a moving point force [4]. 
If $N(\eta), S(\eta)$ are the corrective normal and tangential tractions respectively, the corresponding corrections to the gap opening and slip velocities are

$$
\begin{gathered}
G(\eta)=-\left[\lambda_{2} N(\eta)-\lambda_{1} S(\eta)\right] c_{\nu} J \mu \\
H(\eta)=-\left[\lambda_{3} S(\eta)+\lambda_{1} N(\eta)\right] \frac{c_{t}}{\mu}
\end{gathered}
$$

where

$$
\begin{gathered}
\lambda_{1}=\frac{\mu}{\sin \theta_{1}}\left[\frac{\left(1+2 m_{1} m_{2}-m_{2}^{2}\right)}{\mu R}-\frac{\left(1+2 \bar{m}_{1} \bar{m}_{2}-\tilde{m}_{2}^{2}\right)}{\bar{\mu} \bar{R}}\right], \\
\lambda_{2}=\frac{\mu}{\sin \theta_{1}}\left[\frac{m_{1}\left(1+m_{2}^{2}\right)}{\mu R}+\frac{\bar{m}_{2}\left(1+\bar{m}_{2}^{2}\right)}{\bar{\mu} \bar{R}}\right] \\
\lambda_{3}=\frac{\mu}{\sin \theta_{1}}\left[\frac{m_{2}\left(1+m_{2}^{2}\right)}{\mu R}+\frac{\bar{m}_{2} e\left(1+\bar{m}_{2}^{2}\right)}{\mu \bar{R}}\right]
\end{gathered}
$$

and

$$
\begin{array}{cl}
m_{1}=\cot \theta_{1} ; \quad m_{2}=\cot \theta_{2} ; & \bar{m}_{1}=\cot \theta_{3} ; \quad \bar{m}_{2}=\cot \theta_{4} \\
R=\left(1-m_{2}^{2}\right)^{2}+4 m_{1} m_{2} ; & \bar{R}=\left(1-\bar{m}_{2}^{2}\right)^{2}+4 \bar{m}_{1} \bar{m}_{2} .
\end{array}
$$

The normal traction $N$ is taken positive if tensile, while $S$ is positive when it acts to the right on the lower body. Notice that $G, H$ are influenced only by the local values of $N, S$. The coefficients $\lambda_{2}, \lambda_{3}$ are positive, but $\lambda_{1}$ may be of either sign and vanishes for identical materials.

\section{BOUNDARY CONDITIONS}

If the free surface solution predicts interpenetration (i.e. $g_{0}(\eta)<0$ for some $\eta$ ), we anticipate the development of adhesive or slip contact regions. The tractions in these regions can be determined from physical conditions leading to equalities, e.g. that the gap is zero in a contact zone, whereas the extent of the zones is determined from physical constraints leading to inequalities, e.g. that normal tractions must be non-tensile.

\section{stick}

In a stick zone, the gap is zero and there is no slip. Thus

$$
\begin{aligned}
& \dot{g}(\eta)=G_{0}(\eta)+G(\eta)=0, \\
& \dot{h}(\eta)=H_{0}(\eta)+H(\eta)=0 .
\end{aligned}
$$

Substituting for $G, H$ from (7) and (8) and solving for $S, N$, we find

$$
\begin{aligned}
& S(\eta)=\frac{\mu}{c_{L}}\left[\frac{\lambda_{2} H_{0}-\lambda_{1} G_{0}}{\lambda_{1}{ }^{2}+\lambda_{2} \lambda_{3}}\right] . \\
& N(\eta)=\frac{\mu}{c_{L}}\left[\frac{\lambda_{1} H_{0}+\lambda_{3} G_{0}}{\lambda_{1}{ }^{2}+\lambda_{2} \lambda_{3}}\right] .
\end{aligned}
$$

The permissible extent of the stick zone is determined by the conditions that normal tractions must be non-tensile

$$
N \leqslant 0
$$


and the tangential tractions must lie in the range

$$
|S| \leqslant-f N
$$

wheres is the coefficient of friction. In fact, (18) is included within (19) and can be disregarded. We assume Coulomb friction with equal static and kinetic coefficients.

Substituting (16) and (17) into (19), we find

$$
\left|\lambda_{2} H_{0}-\lambda_{1} G_{0}\right| \leqslant-f\left(\lambda_{1} H_{0}+\lambda_{3} G_{0}\right)
$$

and hence

$$
\left|\lambda_{2} H_{0} \operatorname{sgn} \lambda_{1}-\right| \lambda_{1}\left|G_{0}\right| \leqslant-f\left|\lambda_{1}\right| H_{0} \operatorname{sgn} \lambda_{1}-f \lambda_{3} G_{0}
$$

In view of the absolute value, (21) is equivalent to two inequalities which simplify to

$$
\begin{gathered}
H_{0} \operatorname{sgn} \lambda_{1} \leqslant \gamma_{2} G_{0}, \\
H_{0} \operatorname{sgn} \lambda_{1} \geqslant \gamma_{1} G_{0} ; \quad \gamma_{1}>0, \\
H_{0} \operatorname{sgn} \lambda_{1} \leqslant \gamma_{1} G_{0} ; \quad \gamma_{1}<0,
\end{gathered}
$$

where

$$
\gamma_{1}=\frac{1+\frac{\lambda_{3}}{\left|\lambda_{1}\right|} f}{f-f} ; \quad \gamma_{2}=\frac{1-\frac{\lambda_{3}}{\left|\lambda_{1}\right|} f}{f+f}
$$

and

$$
\hat{f}=\lambda_{2} /\left|\lambda_{1}\right|
$$

Notice that the algebraic sign of $\gamma_{1}$ is the same as that of $(\hat{f}-f)$ which was found to critically influence the nature of the solution in [2]. We also note that $\gamma_{2}$ may be of either sign, but $\left|\gamma_{2}\right| \leqslant\left|\gamma_{1}\right|$. The special case of zero friction corresponds to $\gamma_{1}=\gamma_{2}=1 / \hat{f}$.

$$
\text { SLIP }
$$

In slip zones, the gap is zero and eqn (14) still applies, and hence

$$
\lambda_{2} N-\lambda_{1} S=\mu G_{0} / c_{L}
$$

from (7).

The condition for slip with Coulomb friction is

$$
S=-f N \operatorname{sgn} \dot{h}
$$

We define conforming slip as that for which $\lambda_{1} \dot{h}>0$ and hence

$$
S=-f N \operatorname{sgn} \lambda_{1}
$$

Solving (27) and (29) for $S, N$, we find

$$
\begin{gathered}
S(\eta)=-\frac{\mu f G_{0} \operatorname{sgn} \lambda_{1}}{c_{L}\left(\lambda_{2}+\left|\lambda_{1}\right| f\right)} \\
N(\eta)=\frac{\mu G_{0}}{c_{L}\left(\lambda_{2}+\left|\lambda_{1}\right| f\right)}
\end{gathered}
$$


and the slip velocity is

$$
\dot{h}(\eta)=H_{0}-\gamma_{2} G_{0} \operatorname{sgn} \lambda_{1}
$$

from eqns (8), (30), (31) and (25).

It follows that conforming slip is only permissible if

$$
H_{0} \operatorname{sgn} \lambda_{1}>\gamma_{2} G_{0}
$$

and the requirement of non-tensile tractions imposes the further condition

$$
G_{0} \leqslant 0
$$

from (31).

In non-conforming slip $\lambda_{1} \dot{h}<0$ and we find

$$
\begin{gathered}
S(\eta)=\frac{\mu f G_{0} \operatorname{sgn} \lambda_{1}}{c_{L}\left(\lambda_{2}-\left|\lambda_{1}\right| f\right)} \\
N(\eta)=\frac{\mu G_{0}}{c_{L}\left(\lambda_{2}-\left|\lambda_{1}\right| f\right)} \\
\dot{h}(\eta)=H_{0}-\gamma_{1} G_{0} \operatorname{sgn} \lambda_{1} .
\end{gathered}
$$

Hence, non-conforming slip is only possible if

$$
H_{0} \operatorname{sgn} \gamma_{1}<\gamma_{1} G_{0}
$$

and the requirement $N \leqslant 0$ leads to the further condition

$$
\begin{array}{ll}
G_{0} \leqslant 0 ; & \gamma_{1}>0 ; \\
G_{0} \geqslant 0 ; & \gamma_{1}<0 .
\end{array}
$$

\section{SEPARATION}

In a separation zone there are no corrective tractions and hence the free surface velocities $G_{0}, H_{0}$ are unchanged. The physical condition for separation is that the gap $g(\eta)$ be positive. Notice, however, that when separation starts we must have $G_{0}$ positive. Thus, a transition from contact to separation can only occur when $G_{0} \geqslant 0$ and the reverse transition when $G_{0} \leqslant 0$.

\section{GRAPHICAL REPRESENTATION}

The use of these results in the solution of particular problems is best demonstrated graphically. The first step is to solve the free surface problem for the lower body to find the functions $G_{0}(\eta)$ and $H_{0}(\eta)$. We then plot the expressions $\gamma_{1} G_{0}$ and $\gamma_{2} G_{0}$ as functions of $\eta$ to divide the diagram into regions representing the ranges of the controlling inequalities.

Hence, by plotting the function $H_{0} \operatorname{sgn} \lambda_{1}$ on the same figure we can determine in which regions stick, slip and separation will occur.

$$
\gamma_{1}>0
$$

We first consider the more common case $\gamma_{1}>0$ (see [2]). Figure 2(a) shows $\gamma_{1} G_{0}, \gamma_{2} G_{0}$, $H_{0} \operatorname{sgn} \lambda_{1}$ for a typical example corresponding to the free surface displacement of Fig. 2(b). Notice that if the pulse raises a bulge (as it must do if contact is to occur) the gap opening velocity will be negative on the right side of the bulge and then positive, since the disturbance moves from left to right. 


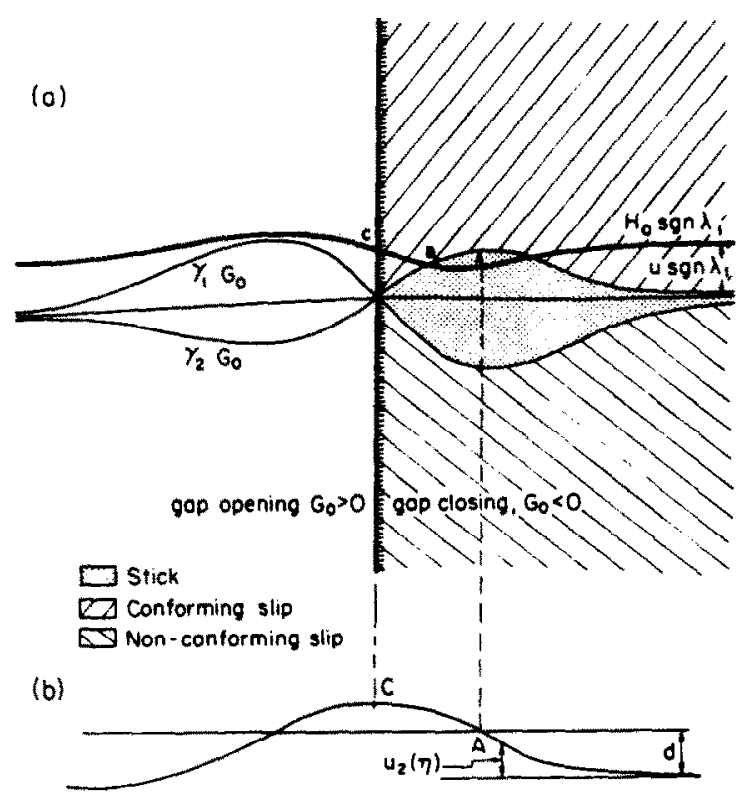

Fig. 2(a). Graphical determination of slip and separation zones for a typical example, $\gamma_{1}>0$. (b) Distortion at a free surface due to the incident pulse of (a).

The diagram is shaded in accordance with the inequalities defining the conditions for stick and slip. For example, stick is only permitted in the range $\gamma_{2} G_{0} \geqslant H_{0} \operatorname{sgn} \lambda_{1} \geqslant \gamma_{1} G_{0}$ and this only exists for $G_{0}<0$ since $\gamma_{1} \geqslant \gamma_{2}$ when $\gamma_{1}>0$. These inequalities define the region between the two lines $\gamma_{2} G_{0}, \gamma_{1} G_{0}$ in the right part of Fig. 2(a) as shown.

For the particular example illustrated we reason as follows:

Contact will not occur to the right of $A$ (Fig. 2b), since the corrective solution has no effect ahead of the disturbance. We identify the corresponding point $A$ in Fig. 2(a) and note that it falls in the stick region, so the contact is initially adhesive and remains so until $B$. Conforming slip commences at $B$ and persists to $C$ where there is a transition to separation.

Notice that for $\gamma_{1}>0$, separation must always start when $G_{0}$ passes through zero i.e. at the maximum point of the free surface bulge-since neither stick nor slip is permitted for $G_{0}<0$.

Away from the pulse, $G_{0}$ tends to zero, but $H_{0}$ tends to the global slip velocity $u$ which is here regarded as a prescribed quantity. The value of $u$ will depend upon the way in which the bodies are supported. The limiting value $u=0$ arises if both bodies are prevented from moving tangentially. The curves for $G_{0}, H_{0}$ are then similar, passing through zero at the same value of $\eta$ and hence the contact will consist of either all slip or all stick. The tangential traction transmitted in the contact zone will generally add up to a tangential force which must be resisted by the support.

Another limiting case of interest is that in which the bodies are unconstrained tangentially so that no net tangential force is transmitted between them. In this case we have to choose $u$ to make the sum of the tangential tractions zero.

If the coefficient of friction $f$ is zero, it follows from eqns (25) that $\gamma_{1}=\gamma_{2}=1 / \hat{f}>0$. The effect on Fig. 2(a) is to make the two lines $\gamma_{1} G_{0}, \gamma_{2} G_{0}$ coincide, eliminating the stick region as we would expect. The solution then proceeds as before. Contact must occur between $A$ and $C$ but it will be either conforming or non-conforming slip depending on the value of $H_{0} \operatorname{sgn} \gamma_{1}$. Separation occurs everywhere to the left of $C$.

$$
\gamma_{1}<0
$$

Figure 3(a,b) shows an example for the case $\gamma_{1}<0 \quad f<\hat{f}$ and $\left.y_{2}>\gamma_{1}\right)$. As in [2], the inequalities are not not mutually exclusive-stick and non-conforming slip are both possible for $H_{0} \operatorname{sgn} \lambda_{1}<\gamma_{1} G_{0}, G_{0}>0$ and the separation point is indeterminate. We emphasize that the solution is not unique in the sense that no physical principles are violated by assuming that separation starts at any arbitrary point to the left of $B$ or indeed that separation never occurs 


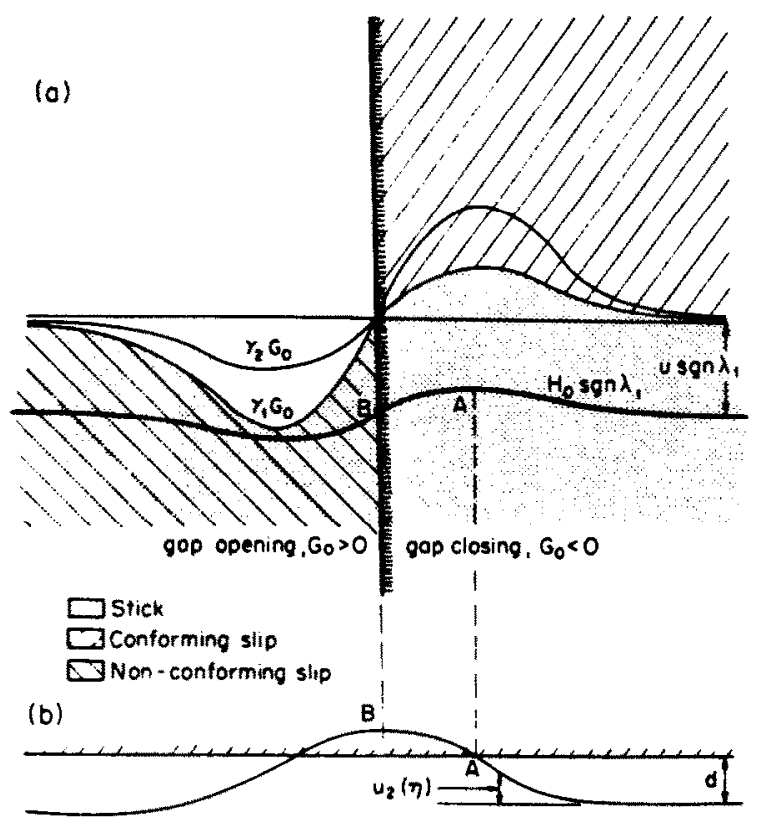

Fig. 3(a). Determination of slip and separation zones for $\gamma_{1}<0$. (b) Free surface distortion for (a).

and the gap remains closed. However, the solution in which separation starts at $B$ might be preferred in that it gives a smoothly opening gap, i.e. the initial value of $\dot{g}$ is zero. Furthermore, if a small irregularity in one of the surfaces causes local separation to the left of $B$, it will be perpetuated, since the gap continues to grow. By contrast, a similar gap developed to the right of $B$ will close again since $G_{0}(\eta)<0$. In this sense, contact to the left of $B$ could be regarded as unstable.

The corrective solution, the extent of the slip and stick zones and the contact tractions depend on the incident pulse only through the functions $G_{0}(\eta)$ and $H_{0}(\eta)$ describing the relative motion of the surfaces in the free surface solution. Such relative motion could be produced by other means-notably if the bodies are curved and roll over each other with microslip so that the contact region moves along the interface at velocity $v$. This problem is treated in a companion paper [5].

Acknowledgement-Support by the National Science Foundation through the grant CME 8019281 is gratefully acknowledged.

\section{REFERENCES}

1. M. Comninou and J. Dundurs, Refiexion and refraction of elastic waves in presence of separation. Proc. $R$. Soc London A356, 509 (1977).

2. M. Comninou, J. R. Barber and J. Dundurs. Disturbance at a frictional interface caused by a plane elastic pulse. $J$. Applied Mechanies, in press.

3. J. D. Achenbach, Wave Propagation in Elastic Solids. North Holland, Amsterdam (1973).

4. A. C. Erineen and E. S. Suhubi, Elastodynamics, Vol. Il, p. 582 . Academic Press, New York (1957).

5. J. R. Barber and M. Comninou, Rolling of elastic cylinders with friction at supersonic speed. Int. J. Solids and Siructures 18, 783 (1982). 